\title{
HELAC - A MONTE CARLO GENERATOR FOR MULTI-JET PROCESSES
}

\author{
C. G. PAPADOPOULOS \\ Institute of Nuclear Physics, NCSR "Demokritos" \\ 15-310 Athens, Greece \\ E-mail: Costas.Papadopoulos@cern.ch \\ M. WOREK * \\ Institute of Nuclear Physics, Polish Academy of Sciences \\ Radzikowskiego 152, 31-342 Krakow, Poland \\ E-mail: Malgorzata.Worek@desy.de
}

\begin{abstract}
The status of the multi-purpose event generator HELAC is briefly presented. The aim of this tool is the full simulation of events within the Standard Model (SM) at current and future high energy experiments, in particular the LHC. Some results related to the production of multi-jet final states at the LHC are also discussed.
\end{abstract}

\section{Introduction}

The possibility of identifying new physics relies on predictions of multi jet final states at current and future collider experiments like TeVatron or LHC. Signals for many models beyond the SM involve a large number of jets resulting from decay chains of particles with high masses. Their Monte Carlo (MC) simulation, both for signal and background is of crucial importance for the success of experiments. One of the ways to simulate multi jet events is to use exact matrix elements at some given order of perturbation theory in the strong coupling constant $\alpha_{s}$. The matrix elements for fully exclusive final states for many jets which we have at our disposal in most cases are at leading order. The advantage of this approach consists of having exact results with all interference effects taken into account properly. The main disadvantage is that more and more diagrams have to be considered when

* Presented at the XIV International Workshop on Deep Inelastic Scattering (DIS2006), Tsukuba, Japan, 20 - 24 April 2006. 
the number of external particles increases. Roughly speaking the number of Feynman diagrams grows asymptotically factorially with the number of external particles.

In this report we will focus on tree level calculations. However, recursive methods based on Dyson-Schwinger recursive equations are used during calculations, resulting in the computational cost of $3^{n}$ comparing to $n$ ! in the traditional Feynman diagrams approach.

\section{Short description of the program}

HELAC is a FORTRAN code which automatically constructs helicity amplitudes using recursion techniques based on Dyson-Schwinger equations. It has been introduced in Ref. [1] and recently revived in Ref. [2-7]. The initial and final state particles are given by the user. Subsequently, the program calculates the corresponding sub-amplitudes which contribute to the process under consideration and evaluates the total amplitude, without referring to individual Feynman diagrams. Apart from that, the summation over helicity and colour configurations, usually time consuming, is performed by MC techniques, see Ref. [7] for details. This results in the computational cost of $3^{n}$, where $n$ is a number of the external particles, compared to $n$ ! in the traditional Feynman diagrams approach. The program is able to calculate the matrix element for any tree level SM process. The phase space sampling subroutines, see Ref. [7] for details, are also implemented to generate total rates as well as partonic events. The program also includes an acceptance-rejection part to unweight those events. The code is able to generate a final state configuration made of hard quarks, gluons and other non-coloured particles. The final state is thus not directly comparable to what is observed in the experiment. An event generator that aspires to give a realistic description of collision processes must include a way to compute/estimate the effects of higher order corrections in perturbation theory and describe hadronisation effects. For parton showering and translation of partons into hadrons the code is interfaced to the latest version of PYTHIA 6.4 [8] in a standard way for FORTRAN based event generators by the Les Houches Accord (LHA) event record [9].

\section{Basic results}

In this section, several numerical results for multi-parton production at the LHC are presented. The main aim is to show that the MC summation over colour, which speeds up the calculation enormously, gives results with 
precision comparable to the one based on explicit summation. The centre of mass energy was chosen to be $\sqrt{s}=14 \mathrm{TeV}$. In order to remain far from collinear and soft singularities and to simulate as much as possible the experimentally relevant phase-space regions, we have chosen the following cuts:

$$
p_{T_{i}}>60 \mathrm{GeV}, \quad\left|y_{i}\right|<2.5, \quad \Delta R_{i j}>1.0
$$

for each pair of outgoing partons $i$ and $j$. All results are obtained with a fixed strong coupling constant $\left(\alpha_{s}=0.13\right)$. For the parton structure functions, we used the CTEQ6 PDF's parametrisation [10,11]. For the phase space generation we used the algorithm described in Ref. [7], whereas in all cases results were cross checked with PHEGAS [12], HAAG [13] and RAMBO [14]. In Tab.1 the results for the total cross section for processes with gluons are presented. All cross sections are in agreement within errors. Rapidity

Table 1. Results for the total cross section for processes with gluons only. $\sigma_{\text {EXACT }}$ corresponds to summation over all possible colour configurations, while $\sigma_{\mathrm{MC}}$ corresponds to MC summation.

$\begin{array}{lrr}\text { Process } & \sigma_{\mathrm{EXACT}} \pm \varepsilon(\mathrm{nb}) & \sigma_{\mathrm{MC}} \pm \varepsilon(\mathrm{nb}) \\ & & \\ g g \rightarrow 2 g & (0.46572 \pm 0.00258) \times 10^{4} & (0.46849 \pm 0.00308) \times 10^{4} \\ g g \rightarrow 3 g & (0.15040 \pm 0.00159) \times 10^{3} & (0.15127 \pm 0.00110) \times 10^{3} \\ g g \rightarrow 4 g & (0.11873 \pm 0.00224) \times 10^{2} & (0.12116 \pm 0.00134) \times 10^{2} \\ g g \rightarrow 5 g & (0.10082 \pm 0.00198) \times 10^{1} & (0.09719 \pm 0.00142) \times 10^{1} \\ g g \rightarrow 6 g & (0.74717 \pm 0.01490) \times 10^{-1} & (0.76652 \pm 0.01862) \times 10^{-1}\end{array}$

and transverse momentum distributions of the most and the least energetic parton for $g g \rightarrow 4 g$ processes are shown in Fig.1 and Fig.2. They clearly demonstrate that MC summation over colour performs very well not only at the level of total rates but also at the level of differential distributions.

\section{Summary}

A status report on an efficient tool for automatic computation of helicity amplitudes and cross sections for multi-jet final states in the SM with the LHA event record interface for parton shower and hadronisation to PYTHIA has been shortly presented. 

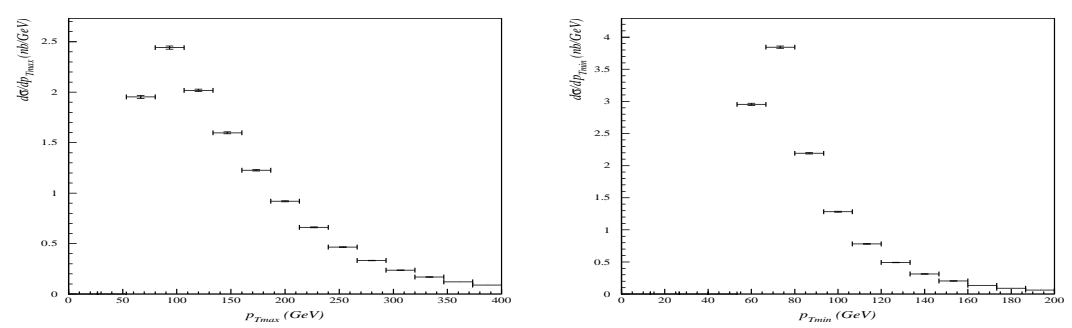

Figure 1. Transverse momentum distribution of the most (left panel) and the least (right panel) energetic gluon in the $g g \rightarrow 4 g$ process with the MC summation over colour.
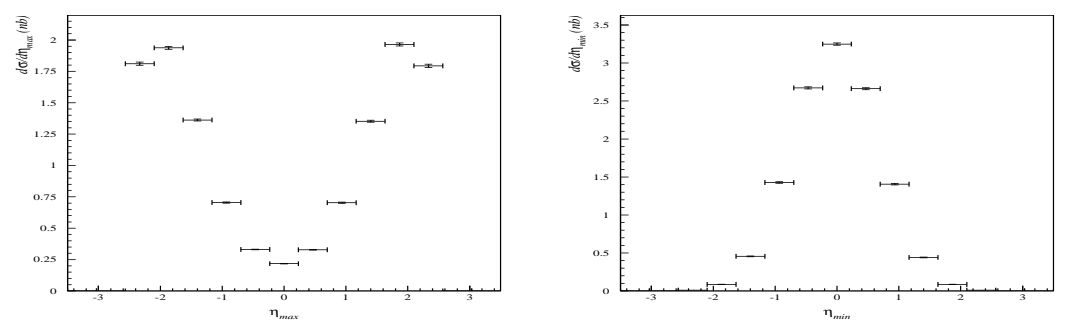

Figure 2. Rapidity distribution of the most (left panel) and the least (right panel) energetic gluon in the $g g \rightarrow 4 g$ process with $M C$ summation over colour.

\section{Acknowledgments}

This work is partly supported by the EU grant MTKD-CT-2004-510126 in partnership with the CERN Physics Department and by the Polish Ministry of Scientific Research and Information Technology grant No 620/E77/6.PR UE/DIE 188/2005-2008. The Greece-Poland bilateral agreement "Advanced computer techniques for theoretical calculations and development of simulation programs for high energy physics experiments" is also acknowledged.

\section{References}

1. P. Draggiotis, R. H. P. Kleiss, and C. G. Papadopoulos, Phys. Lett. B439 (1998) 157-164, hep-ph/9807207.

2. A. Kanaki and C. G. Papadopoulos, Comput. Phys. Commun. 132 (2000) 306-315, hep-ph/0002082.

3. A. Kanaki and C. G. Papadopoulos, Published in AIP Conference Proceed- 
ings - August 20, 2001 - Volume 583, Issue 1, pp. 169-172 and in Workshop On Computer Particle Physics: (CPP 2001): Automatic Calculation For Future Colliders, edited by Y. Kurihara. Tsukuba, Japan, KEK, 2002. 189p. (KEK-PROCEEDINGS-2002-11), p. 20-25, hep-ph/0012004.

4. P. D. Draggiotis, R. H. P. Kleiss, and C. G. Papadopoulos, Eur. Phys. J. C24 (2002) 447-458, hep-ph/0202201.

5. C. G. Papadopoulos and M. Worek, Nucl. Instrum. Meth. A559 (2006) 278281, hep-ph/0508291.

6. C. G. Papadopoulos and M. Worek, Acta Phys. Polon. B36 (2005) 33553367, hep-ph/0510416.

7. C. G. Papadopoulos and M. Worek, hep-ph/0512150.

8. T. Sjostrand, S. Mrenna, and P. Skands, JHEP 05 (2006) 026, hep-ph/0603175.

9. E. Boos et al., hep-ph/0109068.

10. J. Pumplin et al., JHEP 07 (2002) 012, hep-ph/0201195.

11. D. Stump et al., JHEP 10 (2003) 046, hep-ph/0303013.

12. C. G. Papadopoulos, Comput. Phys. Commun. 137 (2001) 247-254, hep-ph/0007335.

13. A. van Hameren and C. G. Papadopoulos, Eur. Phys. J. C25 (2002) 563-574, hep-ph/0204055.

14. R. Kleiss, W. J. Stirling, and S. D. Ellis, Comput. Phys. Commun. 40 (1986) 359. 